\title{
Vasoactive-ventilation-renal score reliably predicts hospital length of stay after surgery for congenital heart disease
}

\author{
Bradley Scherer, MD, ${ }^{a}$ Elizabeth A. S. Moser, MS, ${ }^{b}$ John W. Brown, MD, ${ }^{c}$ Mark D. Rodefeld, MD, ${ }^{c}$ \\ Mark W. Turrentine, MD, ${ }^{\mathrm{c}}$ and Christopher W. Mastropietro, $\mathrm{MD}^{\mathrm{d}}$
}

\begin{abstract}
Objectives: We aimed to further validate the vasoactive-ventilation-renal score as a predictor of outcome in patients recovering from surgery for congenital heart disease. We also sought to determine the optimal time point within the early recovery period at which the vasoactive-ventilation-renal score should be measured.
\end{abstract}

Methods: We prospectively reviewed consecutive patients recovering from cardiac surgery within our intensive care unit between January 2015 and June 2015. The vasoactive-ventilation-renal score was calculated at $6,12,24$, and 48 hours postoperatively as follows: vasoactive-ventilation-renal score $=$ ventilation index + vasoactive-inotrope score $+\Delta$ creatinine [change in serum creatinine from baseline*10]. Primary outcome of interest was prolonged hospital length of stay, defined as length of stay in the upper $25 \%$. Receiver operating characteristic curves were generated, and areas under the curve with $95 \%$ confidence intervals were calculated for all time points. Multivariable logistic regression modeling also was performed.

Results: We reviewed 164 patients with a median age of 9.25 months (interquartile range, 2.6-58 months). Median length of stay was 8 days (interquartile range, 5-17.5 days). The area under the curve value for the vasoactive-ventilation-renal score as a predictor of prolonged length of stay ( $>17.5$ days) was greatest at 12 hours postoperatively (area under the curve $=0.93 ; 95 \%$ confidence interval, 0.89-0.97). On multivariable regression analysis, after adjustment for potential confounders, the 12-hour vasoactive-ventilation-renal score remained a strong predictor of prolonged hospital length of stay (odds ratio, 1.15; 95\% confidence interval, 1.10-1.20).

Conclusions: In a heterogeneous population of patients undergoing surgery for congenital heart disease, the novel vasoactive-ventilation-renal score calculated in the early postoperative recovery period can be a strong predictor of prolonged hospital length of stay. ( $\mathrm{J}$ Thorac Cardiovasc Surg 2016;152:1423-9)

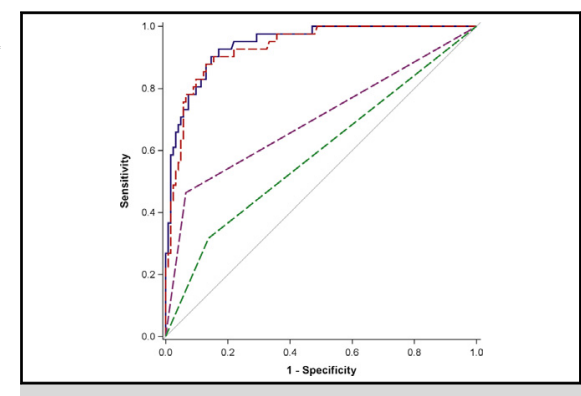

ROC curves for prolonged LOS and the multivariable model (solid) and its individual components (dashed)

\section{Central Message}

The VVR score is a novel measure that robustly predicts outcome after surgery for congenital heart disease.

\section{Perspective}

In this prospective study of patients undergoing surgery for congenital heart disease, the VVR score obtained 12 hours after ICU arrival was a strong predictor of clinical outcome. This novel score could prove to be a powerful tool for bedside assessment of illness severity and prognosis, triage of ICU resources, and stratification of clinical research subjects.

See Editorial Commentary page 1430.

\footnotetext{
From the Departments of ${ }^{\mathrm{a}}$ Pediatrics and ${ }^{\mathrm{c}}$ Cardiac Surgery, and ${ }^{\mathrm{d}}$ Division of Critical Care, Department of Pediatrics, Indiana University School of Medicine, Riley Hospital for Children; and ${ }^{\mathrm{b}}$ Department of Biostatistics, Indiana University School of Medicine \& Richard M. Fairbanks School of Public Health, Indianapolis, Ind.

Indiana University Institutional Review Board Protocol Number 1412965128, approved December 15, 2014, to December 14, 2016.

Received for publication March 16, 2016; revisions received May 24, 2016; accepted for publication July 2, 2016; available ahead of print Sept 17, 2016.

Address for reprints: Christopher W. Mastropietro, MD, Division of Critical Care, Department of Pediatrics, Indiana University School of Medicine, Riley Hospital for Children, 705 Riley Hospital Dr, Indianapolis, IN 46202 (E-mail: cmastrop@ iupui.edu).

$0022-5223 / \$ 36.00$

Copyright (c) 2016 by The American Association for Thoracic Surgery

http://dx.doi.org/10.1016/j.jtcvs.2016.07.070
}

Scoring indices that can accurately reflect the severity of illness in critically ill patients can be extremely valuable in contemporary medicine, providing guidance in patient care (eg, triage, prognostication) and clinical research

Scanning this QR code will take you to supplemental tables and video for this article. 


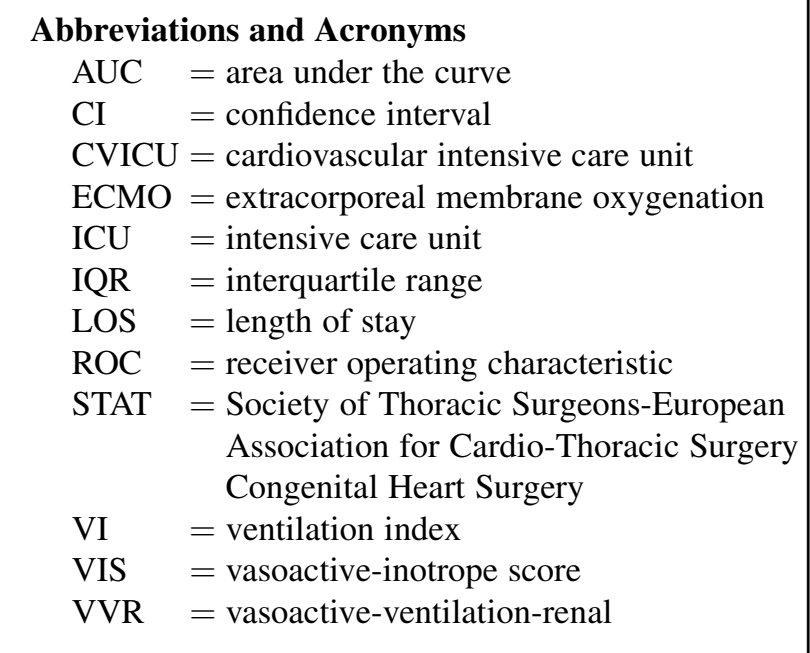

(eg, stratification). However, the development of a disease severity index for children recovering from cardiac surgery has been somewhat elusive, in large part because of the inherent heterogeneity of anatomy and pathophysiology within this patient population. To this end, the novel vasoactive-ventilation-renal (VVR) score has recently demonstrated promise. ${ }^{1,2}$ Specifically, the VVR calculated at 48 hours postoperatively has been shown to be a robust predictor of short-term clinical outcomes and has consistently outperformed the vasoactive inotrope score (VIS) and serum lactate, which have been used as more traditional measures of postoperative disease severity. ${ }^{3-8}$

Our previous studies of the VVR have had notable limitations. These studies have been restricted to patients aged less than 18 years who have required cardiopulmonary bypass. Adults undergoing surgery for congenital heart disease and children undergoing cardiac surgery without cardiopulmonary bypass (eg, systemic-to-pulmonary shunt, pulmonary artery banding) have been excluded. In addition, these prior reports focused only on VVR measurements obtained at admission, peak, and 48-hour measurements. Peak and 48-hour VVR measurements in particular were chosen for these initial studies because these measurements have been the focus of the majority of research on the VIS. ${ }^{3-5}$ The clinical utility of measurements obtained at specific postoperative time points earlier in the postoperative course has not yet been determined. To address these gaps in what is known regarding the VVR, we sought to conduct a more inclusive prospective observational study examining the VVR score. We postulated that the VVR would continue to be more predictive of outcomes compared with the VIS and serum lactate in this broader population of patients recovering from surgery for congenital heart disease. We also sought to determine whether the VVR obtained at distinct time points earlier in the postoperative period, which would have more practical clinical value, would be as predictive of postoperative outcomes as the VVR calculated at 48 hours.

\section{PATIENTS AND METHODS Patient Population}

This prospective observational validation study was approved by the Institutional Review Board within the Office of Research Compliance at Indiana University. Because of the observational nature of the study, consent was waived by the Institutional Review Board. All patients admitted to the cardiovascular intensive care unit (CVICU) at Riley Hospital for Children in Indianapolis, Indiana, from January 1, 2015 , to June 30, 2015, were prospectively reviewed. Patients who required extracorporeal membrane oxygenation (ECMO) for the first 48 hours of CVICU admission were excluded, because their vasoactive medication regimens and ventilator requirements would not be reflective of their underlying disease severity but rather of the mechanical support provided by the extracorporeal circuit. All other patients were followed throughout their clinical courses and included in all analyses.

\section{Data Collection}

Preoperative and perioperative data collected included age, anthropometric measurements at time of surgery, anatomic diagnosis and procedure performed, Society of Thoracic Surgeons-European Association for Cardio-Thoracic Surgery Congenital Heart Surgery (STAT) mortality category, ${ }^{9}$ cardiopulmonary bypass duration, aortic cross-clamp duration, duration of hypothermic circulatory arrest if used, and preoperative serum creatinine values. All arterial blood gas and lactate measurements (which are performed simultaneously using point-of-care testing) obtained during the first 48 postoperative hours were recorded daily by study personnel, along with the ventilator support at the time of each measurement, which included respiratory rate, fraction of inspired oxygen, peak inspiratory pressure, positive end-expiratory pressure, and mean airway pressure. Patients at our institution are typically managed postoperatively with synchronized intermittent mandatory ventilation/pressure-regulated volume control and typically extubated, at the discretion of the primary care team, from mechanical ventilator support when breathing comfortably with good gas exchange and without metabolic acidosis on the following ventilator settings: respiratory rate 10 breaths per minute or less, pressure support $10 \mathrm{~cm} \mathrm{H}_{2} \mathrm{O}$ or less, positive end-expiratory pressure $5 \mathrm{~cm} \mathrm{H}_{2} \mathrm{O}$ or less, and fraction of inspired oxygen concentration 0.4 or less. Doses of inotropic and vasopressor medications (eg, dopamine, dobutamine, epinephrine, norepinephrine, milrinone, and vasopressin) at the time of each arterial blood gas measurement also were recorded. Last, serum creatinine values obtained on admission, postoperative day 1 , and postoperative day 2 were recorded.

\section{Derivation of the Vasoactive Ventilation Renal Score}

Throughout each patient's hospital course, we calculated the patient's VIS at the time of each postoperative arterial blood gas measurement. The VIS was calculated in the following manner ${ }^{3}$ :

$$
\begin{aligned}
\text { VIS }= & \text { dopamine dose }(\mathrm{mcg} / \mathrm{kg} / \mathrm{min})+\text { dobutamine dose } \\
& (\mathrm{mcg} / \mathrm{kg} / \mathrm{min})+100 \times \text { epinephrine dose }(\mathrm{mcg} / \mathrm{kg} / \mathrm{min})+10 \times \\
& \text { milrinone dose }(\mathrm{mcg} / \mathrm{kg} / \mathrm{min})+10,000 \times \text { vasopressin dose }
\end{aligned}
$$$$
(\mathrm{U} / \mathrm{kg} / \mathrm{min})+100 \times \text { norepinephrine dose }(\mathrm{mcg} / \mathrm{kg} / \mathrm{min})
$$ 
For patients on no vasoactive support at the time of blood gas measurement, VIS equaled zero. Second, the ventilation index (VI) was calculated for each postoperative arterial blood gas measurement as follows ${ }^{10}$ :

$$
\mathrm{VI}=(\text { Ventilator RR }) \times(\mathrm{PIP}-\mathrm{PEEP}) \times \mathrm{PaCO}_{2} / 1000
$$

Use of the VI, which incorporates arterial carbon dioxide tension rather than alveolar oxygen tension into its formula, permits the inclusion of patients with mixing lesions and single ventricle anatomy in the study, because arterial carbon dioxide tension is less affected by intracardiac shunting. For patients not requiring mechanical ventilation at the time of arterial blood gas measurement, VI equaled zero. Last, baseline preoperative serum creatinine was subtracted from each postoperative serum creatinine measurement (eg, on admission and postoperative days 1 and 2), which we labeled $\Delta \mathrm{Cr}$. For patients in whom postoperative serum creatinine measurements were less than or equal to baseline, we recorded $\Delta \mathrm{Cr}=0$. Creatinine was measured in $\mathrm{mg} / \mathrm{dL}$. For centers where creatinine is measured in $\mathrm{mmol} / \mathrm{L}$, we recommend converting to $\mathrm{mg} / \mathrm{dL}$ to calculate the score as follows: serum creatinine $(\mathrm{mmol} / \mathrm{dL}) \times 0.0113=$ serum creatinine $(\mathrm{mg} / \mathrm{dL})$.

By using each of these individual measurements, we then calculated the VVR at the time of each arterial blood gas measurement as follows:

$$
\mathrm{VVR}=\mathrm{VIS}+\mathrm{VI}+(\Delta \mathrm{Cr} \times 10)
$$

VVR scores at 6, 12, 24, and 48 hours postoperatively were recorded.

\section{Statistical Analyses}

Descriptive statistics are provided as medians with interquartile ranges (IQRs) for continuous variables and absolute counts with percentages for categoric variables. The primary outcome of interest was hospital length of stay (LOS). We opted to focus the study on hospital LOS because we deemed criteria for hospital discharge compared with intensive care unit (ICU) discharge to likely be less inconsistent across centers. Hospital LOS also is inclusive of time spent in the ICU related to unplanned readmission after transfer to the cardiology ward. This outcome was dichotomized as upper (worst) 25 th percentile versus lower 75 th percentile. Patients in the upper $25 \%$ were defined as having prolonged LOS. Bivariate logistic regression analyses were performed to determine the individual contributions of VVR, VIS, and serum lactate as predictors of LOS. This analysis was repeated for each of the 4 recorded time points. Receiver operative characteristic (ROC) curves were generated, and the abilities of the predictors to correctly classify LOS were compared using area under the curve (AUC) values. AUC values for VVR at the study time points were compared using the method of DeLong and colleagues ${ }^{11}$ as implemented by SAS (SAS Institute Inc, Cary, NC).

Bivariate comparisons were performed for demographic and surgical characteristics of patients with and without prolonged LOS using Wilcoxon rank-sum tests, chi-square test, or Fisher exact test as appropriate for individual variables. Variables that attained a bivariate significance of .30 or less and were of clinical relevance were considered for inclusion in a multivariable logistic regression model. Linearity in the logit was examined for continuous variables before model building; those with evidence of nonlinearity were converted to categoric variables. To obtain the best model, stepwise selection was used with a significance level of .3 for entry into the model and .05 for staying. All statistical analyses were performed using STATA version 13 (StataCorp LP, College Station, Tex) and SAS version 9.4.

\section{RESULTS}

\section{Study Population}

During the study period, 168 unique patients were admitted to the cardiovascular ICU postoperatively.
TABLE 1. Patient demographic characteristics

\begin{tabular}{lc}
\hline \multicolumn{1}{c}{ Characteristic } & All patients $(\mathbf{n}=\mathbf{1 6 4})$ \\
\hline Age (mo) & $9.3(2.6-57.8)$ \\
Male (n) & $102(62 \%)$ \\
Weight $(\mathrm{kg})$ & $7.8(4.6-17.8)$ \\
Race (n) & \\
Caucasian & $131(80 \%)$ \\
African American & $23(14 \%)$ \\
Asian & $7(4 \%)$ \\
Other & $3(2 \%)$ \\
Genetic/chromosomal abnormalities (n) & $35(21 \%)$ \\
Trisomy 21 & $17(10 \%)$ \\
Other & $18(11 \%)$ \\
Noncardiac anatomic abnormalities (n) & $30(18 \%)$ \\
STAT category (n) & \\
1 & $52(32 \%)$ \\
2 & $41(25 \%)$ \\
3 & $31(19 \%)$ \\
4 & $31(19 \%)$ \\
5 & $7(4 \%)$ \\
Other & $2(1 \%)$ \\
Preoperative creatinine (mg/dL) & $0.35(0.29-0.50)$ \\
Cardiopulmonary bypass duration (min) & $88(59-136)$ \\
Aortic crossclamp duration (min) & $42(15-66)$ \\
Delayed sternal closure (n) & $16(10 \%)$ \\
Postoperative inhaled nitric oxide use (n) & $27(16 \%)$ \\
Admission lactate (mg/dL) & $2.0(1.3-2.9)$ \\
Postoperative CPR (n) & $6(4 \%)$ \\
\hline Da & \\
\hline
\end{tabular}

Data represented as absolute counts (\%) or median (IQR). STAT, Society of Thoracic Surgeons-European Association for Cardio-Thoracic Surgery Congenital Heart Surgery; $C P R$, cardiopulmonary resuscitation.

Four patients required ECMO support for the first 48 postoperative hours and were excluded from analysis. All other patients $(\mathrm{N}=164)$ were included in the analysis, with a median age of 9.3 months (range, 1 day to 33 years). A summary of the demographic and clinical characteristics of the patient cohort is shown in Table 1, and a summary of the primary cardiovascular surgical procedures performed in the patient cohort are provided in Table E1. Median hospital LOS, our primary outcome variable, was 8 days (IQR, 5-17.5). Prolonged LOS was defined as greater than 17.5 days. Three patients died before hospital discharge, all of whom had prolonged hospital LOS before their deaths (range, 29-148 days). In addition, the hospital LOS for the 4 excluded patients who required ECMO support for the first 48 hours ranged from 38 to 248 days, and none of these patients died before hospital discharge.

Median serum lactate, VIS, and VVR measurements with IQR and maximum values are presented in Table 2 for all study time points. We obtained ROC curves via bivariate logistic regression analysis for 6-, 12-, 24-, and 48-hour serum lactate, VIS, and VVR as predictors of prolonged LOS. The AUC values for these models also are provided 
TABLE 2. Relationship between postoperative measurements and prolonged length of stay

\begin{tabular}{|c|c|c|c|}
\hline Postoperative variables & Median (IQR) & Maximum & $\operatorname{AUC}(95 \%$ CI $)$ \\
\hline \multicolumn{4}{|l|}{$6 \mathrm{~h}$} \\
\hline Lactate $(\mathrm{mg} / \mathrm{dL})$ & $1.3(0.8-2.6)$ & 9.2 & $0.75(0.67-0.84)$ \\
\hline VIS & $0(0-5)$ & 15.5 & $0.81(0.74-0.88)$ \\
\hline VVR score & $5.2(0.6-24.3)$ & 96 & $0.93(0.89-0.97)$ \\
\hline \multicolumn{4}{|l|}{$12 \mathrm{~h}$} \\
\hline Lactate $(\mathrm{mg} / \mathrm{dL})$ & $1.1(0.7-1.9)$ & 9.7 & $0.78(0.71-0.86)$ \\
\hline VIS & $0(0-5)$ & 16 & $0.81(0.73-0.88)$ \\
\hline VVR score* & $3.5(0.2-24.6)$ & 75 & $0.93(0.89-0.97)$ \\
\hline \multicolumn{4}{|l|}{$24 \mathrm{~h}$} \\
\hline Lactate $(\mathrm{mg} / \mathrm{dL})$ & $0.9(0.7-1.4)$ & 5.4 & $0.61(0.52-0.71)$ \\
\hline VIS & $0(0-5)$ & 17 & $0.84(0.77-0.91)$ \\
\hline VVR score & $1.7(0-20.9)$ & 64 & $0.91(0.84-0.97)$ \\
\hline \multicolumn{4}{|l|}{$48 \mathrm{~h}$} \\
\hline Lactate $(\mathrm{mg} / \mathrm{dL})$ & $0.85(0.6-1.3)$ & 5.4 & $0.55(0.45-0.65)$ \\
\hline VIS & $0(0-4.5)$ & 17.5 & $0.83(0.76-0.91)$ \\
\hline VVR score & $0.5(0-11.1)$ & 64 & $0.89(0.81-0.96)$ \\
\hline Age (mo) & $9.3(2.6-57.8)$ & $33 \mathrm{y}$ & $0.78(0.70-0.87)$ \\
\hline Weight (kg) & $7.8(4.6-17.8)$ & 100 & $0.80(0.72-0.88)$ \\
\hline STAT category & $2(1-3)$ & 5 & $0.79(0.72-0.87)$ \\
\hline CPB duration (min) & $87.5(59-136)$ & 345 & $0.66(0.55-0.77)$ \\
\hline
\end{tabular}

in Table 2. Similar to our prior studies, ${ }^{1,2}$ VVR performed well and had a greater AUC than the corresponding VIS and serum lactate at each time point $(P<.05$ for all comparisons). We also performed ROC analysis for age, weight, duration of cardiopulmonary bypass, and STAT category as predictors of prolonged LOS. The VVR at each of the 4 study time points was more predictive of prolonged LOS than any of these patient variables $(P<.05$ for all comparisons). The ROC curves for prolonged LOS and serum lactate, VIS, and VVR at 12 hours postoperatively, as well as STAT mortality category, are illustrated in Figure 1.

The AUCs for the VVR at 6, 12, 24, and 48 hours are statistically compared with each other in Table E2. There were no statistical differences between the ROC curves for any of the VVR time points. In other words, the VVR at 6,12 , and 24 hours were, at the very least, as predictive as the VVR at 48 hours. Of the 4 VVR measurements, the 12-hour VVR had the highest AUC, 0.93 (95\% confidence interval [CI], 0.89-0.97). For this reason, the remainder of the analysis is focused on this time point.

Demographic and clinical variables for patients with and without prolonged hospital LOS are compared in Table 3. All variables with a $P$ value less than .3 were considered for the multivariable model. On

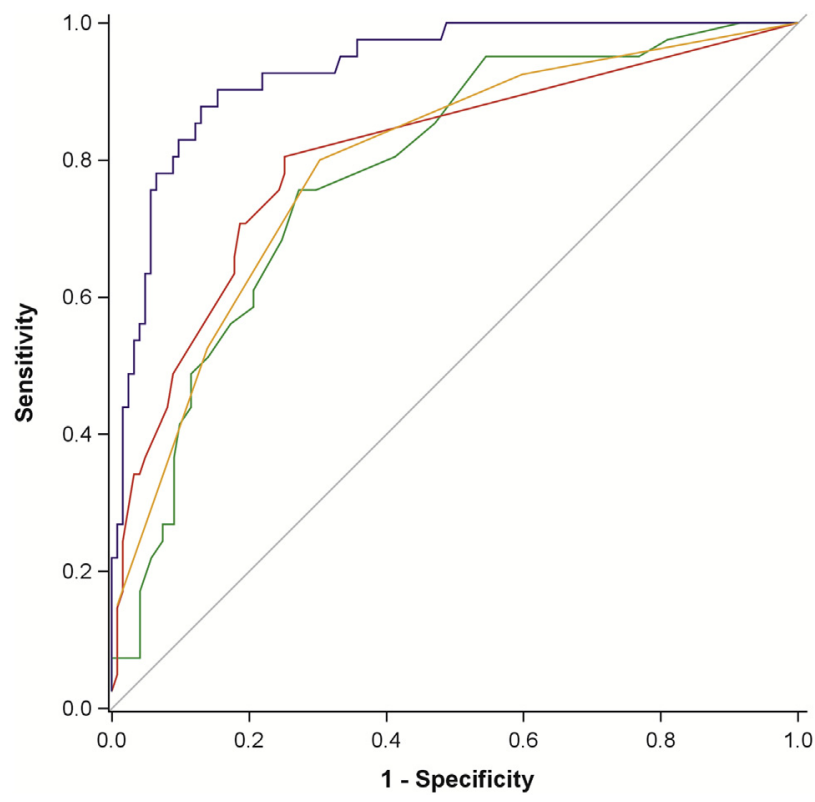

FIGURE 1. ROC curves for prolonged hospital LOS and serum lactate (green), VIS (red), VVR score at 12 hours postoperatively (blue), and STAT category (orange). The AUC was greatest for the VVR at 12 hours postoperatively, $P<.001$ for all comparisons.

multivariable regression analysis, the 12-hour VVR remained a strong independent predictor of prolonged LOS. Specifically, with each increase of 1 in a patient's 12-hour VVR score, the odds of a prolonged LOS increased by $15 \%$ (odds ratio, $1.15 ; 95 \% \mathrm{CI}$, 1.10-1.20). Other variables that were independently associated with prolonged LOS were the presence of noncardiac anatomic abnormalities and the use of inhaled nitric oxide. The remaining variables that were significant on bivariate analysis, namely, age, weight, STAT category 4 or 5 , duration of cardiopulmonary bypass, and admission lactate, were not significant on multivariable analysis and did not appreciably affect the model. Delayed sternal closure and the need for cardiopulmonary resuscitation were not included in the multivariable analysis because of the small number of patients within the study who had these postoperative complications. The best multivariable model for prolonged LOS including the 12hour VVR, inhaled nitric oxide use, and noncardiac anatomic abnormalities is presented in Table 4. The AUC for the model was 0.94 (95\% CI, 0.91-0.98). ROC curves for the model and its individual components are shown in Figure 2.

To simplify the interpretation and use of the 12-hour VVR, we dichotomized the variable into high and low. A 12-hour VVR cutoff value of 25 was chosen to maximize total accuracy and minimize weighted error ratios, 
TABLE 3. Bivariate analysis comparing patients with and without prolonged length of stay

\begin{tabular}{|c|c|c|c|}
\hline Characteristic & Prolonged LOS $(n=41)$ & No prolonged LOS $(n=123)$ & $P$ valu \\
\hline Age (mo) & $1.5(0.3-6.5)$ & $11.5(6.3-80)$ & $<.001$ \\
\hline Male (n) & $28(68 \%)$ & $74(60 \%)$ & .35 \\
\hline Weight $(\mathrm{kg})$ & $4.2(3.4-5.5)$ & $8.5(6.1-22.3)$ & $<.001$ \\
\hline Genetic abnormalities (n) & $7(17 \%)$ & $28(23 \%)$ & .44 \\
\hline NCAA (n) & $13(32 \%)$ & $17(14 \%)$ & .01 \\
\hline STAT category 4 or 5 (n) & $21(51 \%)$ & $17(14 \%)$ & $<.001$ \\
\hline Preoperative creatinine $(\mathrm{mg} / \mathrm{dL})$ & $0.35(0.29-0.48)$ & $0.35(0.29-0.51)$ & .42 \\
\hline $\mathrm{CPB}$ duration (min) & $129(78-173)$ & $82(56-122)$ & .002 \\
\hline Aortic crossclamp duration (min) & $53(22-70)$ & $36(14-60)$ & .13 \\
\hline Delayed sternal closure (n) & $15(37 \%)$ & $1(1 \%)$ & $<.001$ \\
\hline Postoperative iNO use (n) & $19(46 \%)$ & $8(7 \%)$ & $<.001$ \\
\hline Admission lactate $(\mathrm{mg} / \mathrm{dL})$ & $2.3(1.7-5)$ & $1.9(1.3-2.7)$ & .004 \\
\hline VVR at $12 \mathrm{~h}$ & $31(27-40)$ & $1(0-11)$ & $<.001$ \\
\hline
\end{tabular}

Data represented as absolute counts (\%) or median (IQR). LOS, Length of stay; NCAA, noncardiac anatomic abnormalities; STAT, Society of Thoracic Surgeons-European Association for Cardio-Thoracic Surgery Congenital Heart Surgery; $C P B$, cardiopulmonary bypass; $i N O$, inhaled nitric oxide; VVR, vasoactive-ventilation-renal.

and this cutoff correctly classified $90 \%$ of patients. Therefore, patients with 12-hour VVR greater than or equal to 25 were defined as having a high VVR score. On multivariable logistic regression analysis, after adjustment for the presence of inhaled nitric oxide and noncardiac anatomic abnormalities, high VVR at 12 hours remained strongly associated with prolonged LOS (odds ratio, 31; 95\% CI, 10-90).

\section{DISCUSSION}

We have further validated the VVR as a multiorgan system severity of illness index for patients recovering from surgery for congenital heart disease. (See Video 1 for additional discussion.) We have now demonstrated that the VVR strongly predicts hospital LOS, more so than the VIS and serum lactate, in a patient population who not only includes children requiring cardiopulmonary bypass but also children undergoing procedures without cardiopulmonary bypass and adults with congenital heart disease. The patients included in this study reflect the postoperative patient populations at many centers, where adults undergoing surgery for congenital heart disease are commonly cared for within pediatric cardiovascular

TABLE 4. Multivariable regression analysis for predictors of prolonged length of stay

\begin{tabular}{lcccc}
\hline \multicolumn{1}{c}{ Parameter } & Estimate & SE & $\boldsymbol{P}$ value & OR $(\mathbf{9 5} \% \mathbf{C I})$ \\
\hline Intercept & -4.20 & 0.64 & $<.0001$ & - \\
12-h VVR score & 0.14 & 0.02 & $<.0001$ & $1.15(1.10-1.20)$ \\
Inhaled nitric oxide & 1.50 & 0.71 & .033 & $4.50(1.13-17.99)$ \\
Noncardiac anatomic & 1.54 & 0.73 & .034 & $4.67(1.13-19.34)$ \\
$\quad$ abnormalities & & & & \\
\hline
\end{tabular}

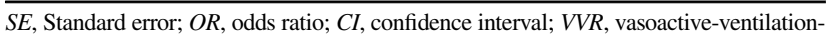
renal.
ICUs. ${ }^{12,13}$ The greater heterogeneity of the patient population in this study has increased the strength and practicality of the VVR.

In our prior work, the VVR calculated at 48 hours was superior to admission and peak VVR measurements. ${ }^{1,2}$ In fact, admission measurements performed poorly. This finding was not surprising, because vasoactive and ventilator support on CVICU admission are likely less reflective of organ dysfunction and illness severity but rather more

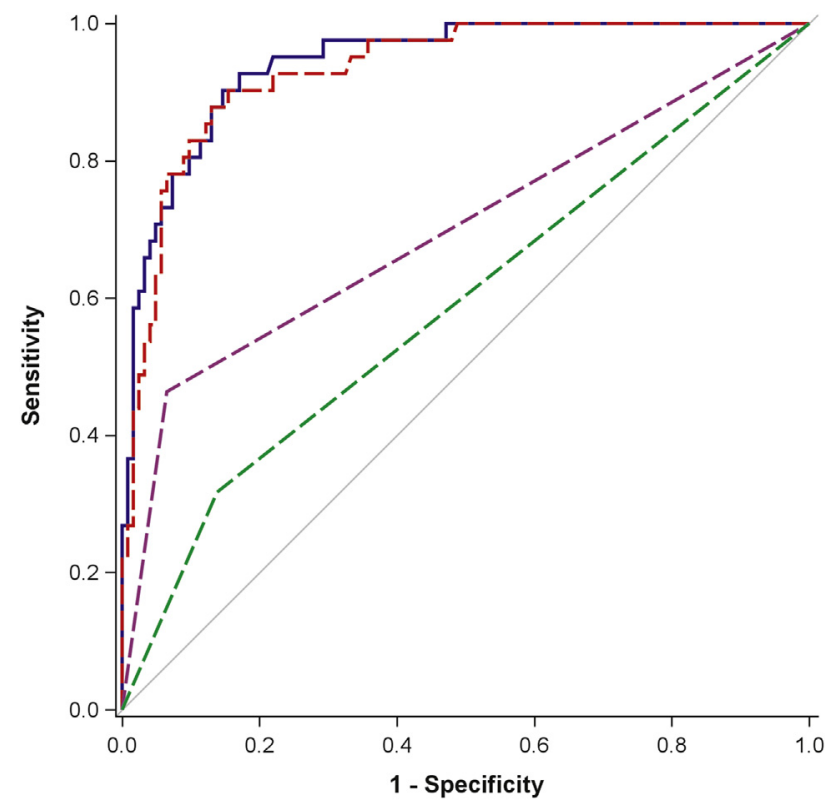

FIGURE 2. ROC curves for prolonged hospital LOS and the multivariable model (solid blue line) and its individual components (dashed lines): VVR score at 12 hours postoperatively (red), inhaled nitric oxide (purple), and noncardiac anatomic abnormalities (green). AUC for the multivariable model was 0.94 (95\% CI, 0.91-0.98). 


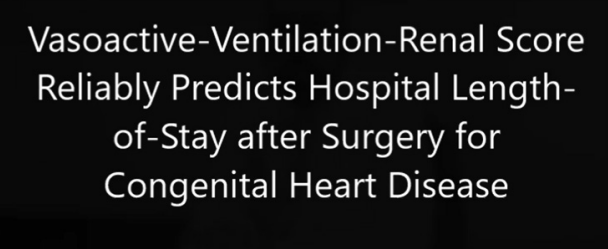

VIDEO 1. Dr Mastropietro explains the inception of the VVR score as an index of disease severity for infants and children who undergo cardiovascular surgery, and comments on the significance of the findings presented in their latest study. Video available at: http://www.jtcvsonline.org/article/ S0022-5223(16)30943-6/addons.

related to the dynamic processes that occur after cessation of cardiopulmonary bypass and during transport to the CVICU. Likewise, the support on admission also may be the peak support, which will negatively affect the strength of peak VVR measurements. We have now demonstrated that the VVR calculated at specific time points earlier than 48 hours postoperatively can reliably predict clinical outcomes in children recovering from cardiac surgery. Specifically, patients with a VVR score greater than or equal to 25 at 12 hours postoperatively were 31 times more likely to have a prolonged LOS compared with patients with lower VVR scores. This ability to provide important prognostic data earlier in the postoperative course further increases the functionality of the VVR.

The advantages of the VVR compared with the VIS and serum lactate, as we have discussed in our prior work, ${ }^{1,2}$ should be intuitive to physicians caring for these children, because the VVR likely captures that subset of patients who have may have preserved hemodynamic integrity yet have significant burden of disease from postoperative lung or kidney injury. The additional components of the VVR, although on the surface may seem cumbersome to some, can be obtained from routinely available bedside data, and the score can be quantitated with a simple calculator. Incorporation of VVR calculation into the electronic medical record could further enhance its utility. If available to physicians at the bedside, the 12-hour VVR has several potential clinical applications. The 12-hour VVR could provide a reliable estimate of illness severity, give parents and families realistic expectations of their child's postoperative course, or assist with triage CVICU resources. In addition, the 12-hour VVR could help stratify potential research subjects or assist with propensity score matching. Further validation in a multicenter data set should be performed before widespread application is recommended.

This study also found that the use of inhaled nitric oxide and the presence of noncardiac anatomic abnormalities had an effect on hospital LOS that was independent of the organ dysfunction quantitated by the 12-hour VVR. Use of inhaled nitric oxide, in many cases, represents an escalation of cardiopulmonary support beyond traditional vasoactive medications and ventilatory support. Therefore, it is somewhat intuitive that its use is associated with prolonged LOS, independently of the VVR. Likewise, the presence of noncardiac anatomic abnormalities such as airway anomalies or gastrointestinal defects (eg, intestinal malrotation, imperforate anus, Hirschsprung's disease) would not be accounted for by the VVR yet could surely prolong LOS, especially if surgical interventions were needed to address those abnormalities. Although the VVR at 12 hours in and of itself was a solid predictor of prolonged LOS, addition of these important variables further elevated the strength of our predictive model (Figure 2).

\section{Study Limitations}

These data come from a single center, which limits their generalizability. However, the initial work on the VVR was completed at a different center, ${ }^{1,2}$ and thus the VVR has now been validated as a predictor of postoperative outcome at 2 different institutions with different cardiac surgeons and varying practices. A multicenter validation study is the next step. Larger studies also should determine whether the VVR has the ability to predict additional outcomes (most important of which is postoperative mortality) and whether other variables such as delayed sternal closure or the need for cardiopulmonary resuscitation have an effect on outcome independently of the VVR. We acknowledge that the VVR cannot reliably be calculated for patients requiring ECMO support, although the need for ECMO in these patients, in and of itself, is considered by most clinicians to be a reliable marker of disease severity. Indeed, all 4 patients who were excluded because of ECMO support would be characterized as having prolonged LOS. Also, no patients in our cohort required peritoneal dialysis or continuous renal replacement therapy within the first 48 hours postoperatively, but either modality could affect the reliability of the score. Finally, the VVR remains affected by limitations previously noted in the $\mathrm{VIS}^{3}$; it does not assess the importance of the individual components of the VIS, and vasoactive medication administration and the degree of mechanical ventilator support were not under protocol and so may have been affected by variation in physician practice.

\section{CONCLUSIONS}

We have further validated the utility of the VVR score after surgery for congenital heart disease. The VVR 
calculated at 12 hours postoperatively could be a valuable and potentially powerful clinical tool to predict important postoperative outcomes in this patient population.

\section{Conflict of Interest Statement}

Authors have nothing to disclose with regard to commercial support.

\section{References}

1. Miletic KG, Spiering TJ, Delius RE, Walters HL III, Mastropietro CW. Use of a novel vasoactive-ventilation-renal score to predict outcomes after paediatric cardiac surgery. Interact Cardiovasc Thorac Surg. 2015;20: 289-95.

2. Miletic KG, Delius RE, Walters HL III, Mastropietro CW. Prospective validation of a novel vasoactive-ventilation-renal score as a predictor of outcomes after pediatric cardiac surgery. Ann Thorac Surg. 2016;101: 1558-63.

3. Gaies MG, Jeffries HE, Niebler RA, Pasquali SK, Donohue JE, Yu S, et al. Vasoactive-inotropic score is associated with outcome after infant cardiac surgery: an analysis from the Pediatric Cardiac Critical Care Consortium and Virtual PICU System Registries. Pediatr Crit Care Med. 2014; 15:529-37.

4. Davidson J, Tong S, Hancock H, Hauck A, da Cruz E, Kaufman J. Prospective validation of the vasoactive-inotropic score and correlation to short-term outcomes in neonates and infants after cardiothoracic surgery. Intensive Care Med. 2012;38:1184-90.

5. Kalyanaraman M, DeCampli WM, Campbell AI, Bhalala U, Harmon TG, Sandiford P, et al. Serial blood lactate levels as a predictor of mortality in children after cardiopulmonary bypass surgery. Pediatr Crit Care Med. 2008; 9:285-8.
6. Crow SS, Robinson JA, Burkhart HM, Dearani JA, Golden AW. Duration and magnitude of vasopressor support predicts poor outcome after infant cardiac operations. Ann Thorac Surg. 2014;98:655-61.

7. Friedland-Little JM, Hirsch-Romano JC, Yu S, Donohue JE, Canada CE, Soraya $\mathrm{P}$, et al. Risk factors for requiring extracorporeal membrane oxygenation support after a Norwood operation. J Thorac Cardiovasc Surg. 2014; 148:266-72.

8. Esch JJ, Salvin JM, Thiagarajan RR, del Nido PJ, Rajagopal SK. Acute kidney injury after Fontan completion: Risk factors and outcomes. J Thorac Cardiovasc Surg. 2015;150:190-7.

9. Jacobs JP, O'Brien SM, Pasquali SK, Jacobs ML, Lacour-Gayet FG, Tchervenkov CI, et al. Variation in outcomes for risk-stratified pediatric cardiac surgical operations: an analysis of the STS Congenital Heart Surgery Database. Ann Thorac Surg. 2012;94:564-71.

10. Paret G, Ziv T, Barzilai A, Ben-Abraham R, Vardi A, Manisterski Y, et al Ventilation index and outcome in children with acute respiratory distress syndrome. Pediatr Pulmonol. 1998;26:125-8.

11. DeLong ER, DeLong DM, Clarke-Pearson DL. Comparing the areas under two or more correlated receiver operating characteristic curves: a nonparametric approach. Biometrics. 1988;44:837-45.

12. Mahle WT, Kirshbom PM, Kanter KR, Kogon BM. Cardiac surgery in adults performed at children's hospitals: trends and outcomes. J Thorac Cardiovasc Surg. 2008;136:307-11.

13. May LJ, Longhurst CA, Pageler NM, Wood MS, Sharek PJ, Zebrack CM. Optimizing care of adults with congenital heart disease in a pediatric cardiovascular ICU using electronic clinical decision support. Pediatr Crit Care Med. 2014;15:428-34.

Key Words: heart defects, congenital, postoperative care, assessment, patient outcomes, neonatal, intensive care units, pediatric, surgery, cardiac 
TABLE E1. Cardiovascular surgical procedures organized by STAT mortality categories

\begin{tabular}{|c|c|}
\hline Primary surgical procedure & $\begin{array}{c}\text { All patients } \\
(\mathrm{N}=164)\end{array}$ \\
\hline STAT category 1 & $52(32 \%)$ \\
\hline Repair, atrial septal defect & 9 \\
\hline Repair, ventricular septal defect & 13 \\
\hline Repair, tetralogy of Fallot & 9 \\
\hline Pulmonary valve replacement & 7 \\
\hline Lateral tunnel Fontan operation & 4 \\
\hline Repair, partial atrioventricular canal defect & 3 \\
\hline Other & 7 \\
\hline STAT category 2 & $41(25 \%)$ \\
\hline Repair, coarctation of the aorta & 8 \\
\hline Aortic valvuloplasty & 6 \\
\hline Repair, tetralogy of Fallot & 4 \\
\hline Extracardiac Fontan operation & 3 \\
\hline Bidirectional Glenn operation & 3 \\
\hline Other & 17 \\
\hline STAT category 3 & $31(18 \%)$ \\
\hline Arterial switch procedure (d-TGA w/IVS) & 5 \\
\hline Hemi-Fontan operation & 6 \\
\hline AV canal & 5 \\
\hline Repair, pulmonary atresia w/VSD & 5 \\
\hline Right ventricle-to-pulmonary artery conduit & 4 \\
\hline Other & 6 \\
\hline STAT category 4 & $31(19 \%)$ \\
\hline Systemic-to-pulmonary artery shunt & 7 \\
\hline Orthotopic heart transplant & 5 \\
\hline Repair, total anomalous pulmonary venous return & 5 \\
\hline Arterial switch procedure (d-TGA w/VSD) & 2 \\
\hline Pulmonary artery banding & 3 \\
\hline Other & 9 \\
\hline STAT category 5 & $7(4 \%)$ \\
\hline Norwood operation & 6 \\
\hline Damus-Kaye-Stansel operation & 1 \\
\hline
\end{tabular}

TABLE E2. Statistical comparison for area under the curve values for the vasoactive-ventilation-renal score and prolonged length of stay at 6 , 12 , 24, and 48 hours

\begin{tabular}{lccccc}
\hline Contrast & Estimate & SE & $\mathbf{9 5} \%$ Wald CL & Chi-square & $\boldsymbol{P}$ value \\
\hline VVR 6 h - VVR 12 h & -0.00366 & 0.0105 & -0.0242 & 0.0169 & 0.1213 \\
VVR 6 h - VVR 24 h & 0.0210 & 0.0252 & -0.0283 & 0.0704 & .73 \\
VVR 6 h - VVR 48 h & 0.0403 & 0.0280 & -0.0145 & 0.0952 & .6978 \\
VVR 1 h - VVR 24 h & 0.0247 & 0.0232 & -0.0208 & 0.0702 & .0787 \\
VVR 1 2 h - VVR 48 h & 0.0440 & 0.0282 & -0.0112 & 0.0992 & 1.1335 \\
VVR 24 h - VVR 48 h & 0.0193 & 0.0197 & -0.0192 & 0.0578 & 2.4421 \\
\hline
\end{tabular}

$S E$, Standard error; $C L$, confidence limit; $V V R$, vasoactive-ventilation-renal score. 